\title{
Pacientes com Transtorno do Espectro Autista e desafio para atendimento odontológico - revisão de literatura
}

Patients with Autistic Spectrum Disorder and challenge for dental care - literature review

Pacientes con Trastorno del Espectro Autista y desafío para el cuidado dental - revisión de la

literatura

Recebido: 26/10/2021 | Revisado: 04/11/2021 | Aceito: 07/11/2021 | Publicado: 11/11/2021

\author{
Fernanda Santos Araujo \\ ORCID: https://orcid.org/0000-0002-7459-6957 \\ Universidade Federal de Sergipe, Brasil \\ E-mail: fsa.odontologia@gmail.com \\ Cristiano Gaujac \\ ORCID: https://orcid.org/0000-0002-4808-9468 \\ Universidade Federal de Sergipe, Brasil \\ E-mail: cgaujac@gmail.com \\ Cleverson Luciano Trento \\ ORCID: https://orcid.org/0000-0002-1079-4217 \\ Universidade Federal de Sergipe, Brasil \\ E-mail: cleverson@ academico.ufs.br \\ Regiane Cristina do Amaral \\ ORCID: https://orcid.org/0000-0002-9191-0960 \\ Universidade Federal de Sergipe, Brasil \\ E-mail: amaralre@yahoo.com.br
}

\begin{abstract}
Resumo
O transtorno do espectro autista (TEA) caracteriza-se pelos déficits na comunicação, na linguagem e nos padrões de comportamentos restrito e repetitivo. A frequência de indivíduos diagnosticados com TEA tem aumentado no decorrer dos últimos anos, e consequentemente leva a uma maior demanda de pacientes nos consultórios odontológicos. Este aumento da demanda faz com que o profissional cirurgião dentista necessite de crtg5bonhecimento acerca das particularidades do paciente com TEA, comorbidades associadas e patologias orais mais comuns, para realizar uma conduta terapêutica eficaz e devolver saúde ao paciente. Assim, o presente estudo teve como objetivo realizar uma revisão de literatura sobre a importância do tratamento odontológico no paciente com transtorno do espectro autista por meio da análise dos acometimentos bucais mais comuns nesses indivíduos, bem como dos desafios enfrentados pelo cirurgião-dentista frente a toda complexidade do TEA. Como metodologia para o estudo foi realizada busca por palavraschave autismo, odontologia, saúde bucal e odontopediatria nas bases de dados Lilacs, Pubmed e Scielo. Conclusão: diante das inúmeras dificuldades encontradas pelos pais e cuidadores, os pacientes portadores do transtorno do espectro autista devem receber um tratamento interdisciplinar, onde o cirurgião-dentista pertencente a esta equipe deve transmitir a importância dos cuidados preventivos em relação as patologias bucais e as orientações quanto à dieta e higiene bucal, bem como o tratamento adequado.
\end{abstract}

Palavras-chave: Transtorno do Espectro Autista; Odontologia; Saúde bucal; Odontopediatria.

\begin{abstract}
Autism spectrum disorder (ASD) is characterized by deficits in communication, language, and restricted and repetitive behavior patterns. The frequency of individuals diagnosed with ASD has increased over the past few years, and consequently leads to a greater demand for patients in dental offices. This increased demand makes the dental surgeon need knowledge about the particularities of the patient with ASD, associated comorbidities and more common oral pathologies, in order to carry out an effective therapeutic approach and restore the patient's health. Thus, this study aimed to carry out a literature review on the importance of dental treatment in patients with autism spectrum disorder through the analysis of the most common oral affections in these individuals, as well as the challenges faced by the dentist in the face of all. complexity of the TEA. As a methodology for the study, a search was performed for the keywords autism, dentistry, oral health and pediatric dentistry in the Lilacs, Pubmed and Scielo databases. Conclusion: given the numerous difficulties encountered by parents and caregivers, patients with autism spectrum disorder must receive an interdisciplinary treatment, where the dentist belonging to this team must convey the importance of preventive care in relation to oral pathologies and guidelines regarding diet and oral hygiene, as well as proper treatment.
\end{abstract}

Keywords: Autism Spectrum Disorder; Dentistry; Oral health; Pediatric dentistry. 


\begin{abstract}
Resumen
El trastorno del espectro autista (TEA) se caracteriza por deficiencias en la comunicación, el lenguaje y patrones de comportamiento restringidos y repetitivos. La frecuencia de personas diagnosticadas con TEA ha aumentado en los últimos años y, en consecuencia, conduce a una mayor demanda de pacientes en los consultorios dentales. Esta mayor demanda hace que el cirujano dentista necesite conocer las particularidades del paciente con TEA, comorbilidades asociadas y patologías bucales más frecuentes, con el fin de llevar a cabo un abordaje terapéutico eficaz y restaurar la salud del paciente. Así, el presente estudio tuvo como objetivo realizar una revisión de la literatura sobre la importancia del tratamiento odontológico en pacientes con trastorno del espectro autista a través del análisis de las afecciones bucales más comunes en estos individuos, así como los desafíos que enfrenta el odontólogo ante la toda la complejidad de la TEA. Como metodología del estudio, se realizó una búsqueda de las palabras clave autismo, odontología, salud bucal y odontología pediátrica en las bases de datos Lilacs, Pubmed y Scielo. Conclusión: dadas las numerosas dificultades que encuentran los padres y cuidadores, los pacientes con trastorno del espectro autista deben recibir un tratamiento interdisciplinario, donde el odontólogo perteneciente a este equipo debe transmitir la importancia de los cuidados preventivos en relación a las patologías bucales y las pautas en materia de alimentación e higiene bucal. así como un tratamiento adecuado.
\end{abstract}

Palabras clave: Trastorno del Espectro Autista; Odontología; Salud bucal; Odontología pediátrica.

\title{
1. Introdução
}

O autismo, também nomeado de transtorno do espectro autista (TEA) é considerado uma síndrome comportamental de neuro-desenvolvimento. Caracteriza-se por alterações dos padrões de comportamento, combinados com a dificuldade de comunicação e interação social (Gomes et al., 2015; Kessamiguiemon et al., 2017). O TEA manifesta-se na primeira infância e acomete principalmente crianças do sexo masculino e uma vez presente, o transtorno permanece no indivíduo durante toda a vida. O fato da criança apresentar o transtorno, não significa que ela necessariamente tenha um déficit cognitivo (Volpato et al., 2013; Tarelho., 2005; Amaral et al., 2012).

Não há uma causa específica para desencadear esse transtorno. Acredita-se que seja de etiologia múltipla, associada a fatores ambientais, neurobiológicos e genéticos, e compromete o processo de desenvolvimento da criança. O autismo pode acontecer de forma isolada ou em combinação com outros distúrbios mentais e normalmente é diagnosticado por psicólogos e psiquiatras (França., 2017; Sant'Anna et al., 2017; APA., 2014).

A frequência de indivíduos diagnosticados com TEA tem aumentado no decorrer dos últimos anos, e consequentemente leva a uma maior demanda de pacientes nos consultórios odontológicos. Isto traz a necessidade do preparo do dentista para atender estes pacientes, que por apresentarem necessidades especiais, geralmente possuem higiene bucal deficiente, devido ao déficit motor, sensorial e cognitivo. Com isso o cirurgião dentista torna-se aliado dos pacientes e cuidadores para promover uma saúde bucal adequada (Sant'Anna et al., 2017; Schimidt et al., 2007; Oredugba \& Akindayomi., 2008)

Devido aos indivíduos portadores do espectro autista possuírem alteração na percepção sensorial, o uso de técnicas humanizadas e consultas com tempo reduzido ajudam no atendimento odontológico. Além desses fatores a utilização de cartões de imagens ilustrativas e aplicativos móveis também influenciam positivamente nas consultas. (Zink et al., 2016; Fakhruddin \& Batawi., 2017; Busato et al., 2017).

Esta revisão de literatura tem o objetivo de salientar a importância do tratamento odontológico em pacientes que possuem TEA e os desafios enfrentados pelos profissionais da odontologia. Além disso, são ressaltadas as estratégias utilizadas pelos dentistas com a finalidade de otimizar a qualidade do atendimento aos portadores do espectro autista, bem como instruir os pais e cuidadores.

\section{Metodologia}

Esse trabalho consiste em uma revisão de literatura que teve como base o estudo de trabalhos acadêmicos, incluindo artigos científicos. Para tanto, foram utilizadas as plataformas Pubmed, Lilacs e SciELO para a pesquisa dos artigos científicos, utilizando-se as palavras chave: Autismo, Odontologia, Saúde Bucal, Odontopediatria. 
Esse trabalho consiste em uma revisão de literatura narrativa que teve como base fontes bibliográficas e eletrônicas, desde trabalhos acadêmicos à artigos científicos entre os anos 1943 e 2020. Para tanto, foram utilizadas as plataformas Pubmed, Lilacs e SciELO para a pesquisa dos artigos científicos, utilizando-se as palavras chave: Autismo, Odontologia, Saúde Bucal, Odontopediatria.

\section{Revisão de Literatura}

\section{Transtorno do espectro autista}

O transtorno do espectro autista foi delineado primordialmente por um renomado psiquiatra pediátrico, Leo Kanner, em 1943. Em seu artigo foi relatado o comportamento diferenciado de um grupo de crianças, onde acreditava que elas podiam apresentar algum traço psicológico que impedia contatos sociais habituais, dificultando o convívio com outras pessoas (Zink et al., 2018; Udhya et al., 2014; Kanner., 1943).

Este transtorno é uma síndrome de neuro-desenvolvimento, de base biológica, descrita como um distúrbio incapacitante do desenvolvimento mental e emocional e pode afetar crianças de qualquer etnia e classe social (Mello., 2007; Souza et al., 2017; APA., 2013; Predebon., 2013). É caracterizada por dificuldade de interação social, distúrbios de linguagem e deficiência cognitiva, que gera padrões de comportamento estereotipados (Santos \& Fronza., 2011). Hábitos como fala repetitiva (ecolalia), alinhar ou girar objetos sem finalidade específica, adesão inflexível as rotinas (criação de "rituais" em atividades diárias) são observadas em pacientes portadores do TEA (Sant'Anna et al., 2017).

Apesar do espectro autista ser definido como um transtorno de desenvolvimento, pelo fato dos sintomas iniciais aparecerem nos primeiros anos de vida, ele é frequentemente considerado como um transtorno vitalício por acarretar consequências negativas em diversas áreas como desempenho escolar, profissional, econômico e na qualidade de vida (Baronio et al., 2015; Dell'Osso., 2015).

O diagnóstico deste transtorno é realizado através da observação de aspectos clínicos, tendo como base características comportamentais e informações relatadas por pais e/ou cuidadores (Gomes et al., 2015). Segundo a American Psychiatry Association (APA.,2014), o TEA manifesta-se de formas variáveis com diferentes sintomas e graus de severidade, justificando assim o termo espectro.

A etiologia do TEA é uma grande incógnita para a ciência. Alguns autores consideram como desconhecida (Dell'Osso., 2018), e outros relatam ser multifatorial, associada a fatores genéticos e neurobiológicos (Gomes et al., 2015; Weddell., 2011). É importante investigar e entender os aspectos no qual o indivíduo está inserido, pois acredita-se que a etiologia do transtorno do espectro autista esteja ligada a diversos fatores ambientais, genéticos e fisiológicos, que sugerem o aumento da ocorrência de TEA na infância (Santos \& Fronza., 2011)..

Em nível mundial a prevalência de indivíduos diagnosticados com TEA é altíssima, estimando-se que 1 em cada 160 crianças apresentam esta condição (Bosa., 2006). O CDC (Centro de Controle de Doenças e Prevenção do governo dos EUA) apresentou um estudo onde a prevalência é de 1 em cada 54 crianças de 8 anos, representando um acréscimo de $10 \%$ do estudo anterior realizado em 2014. A proporção do número de diagnósticos de meninos permanece 4 vezes maior que o de meninas (Mello., 2007; Arvidsson et al., 2018; Baio et al, 2018). Em contrapartida, meninas tendem a ser mais seriamente afetadas e com maior comprometimento cognitivo (Tarelho., 2005). No Brasil, segundo estimativas globais da ONU, 2 milhões de indivíduos possuem TEA, constituindo aproximadamente 1\% da população (Maenner et al., 2020).

\section{Importância do tratamento odontológico no paciente com transtorno do espectro autista}

Geralmente o primeiro contato do paciente portador de TEA ocorre de forma tardia. Os responsáveis, diante às dificuldades em realizar higiene bucal em casa e devido à falta de cooperação da criança, demoram a levar o paciente ao 
ambulatório. Por demandar tempo para conseguir a confiança do paciente, normalmente, não se consegue êxito na primeira consulta. Por isso, inicialmente, o dentista deve condicionar o paciente e colher informações dos responsáveis através de uma minuciosa anamnese. Além de informar aos pais e /ou cuidadores sobre a importância do cuidado com a higiene bucal e ensinar as técnicas para que eles possam reproduzir em casa (França., 2017; Oliveira., 2018).

A comunicação entre o paciente e o dentista representa uma dificuldade durante o atendimento odontológico, principalmente quando se trata de pacientes com déficits sensoriais, pois estes pacientes geralmente apresentam dificuldade de socialização, medo e ansiedade exacerbados (Oliveira., 2018). Desse modo, o profissional deve ser capacitado e ter o conhecimento necessário sobre técnicas de manejo individualizadas considerando o grau do transtorno e as patologias presentes na cavidade oral, a fim de facilitar e otimizar os procedimentos nestes pacientes (Amaral et al., 2011; Villar et al., 2016).

Segundo alguns autores, devido ao grau do transtorno, alguns pacientes podem ser incapazes de tolerar procedimentos no consultório odontológico, sendo necessária a realização do procedimento sob anestesia geral em ambiente hospitalar. Isso oferece uma maior eficiência nos cuidados aos pacientes não cooperativos (Fonseca et al., 2010; Mathu-Muju et al., 2016; Perez et al., 2016).

\section{Saúde bucal do paciente com transtorno do espectro autista}

Pacientes portadores de TEA não apresentam problemas de saúde bucal específicos à doença, mas é esperado que nesses pacientes o risco à cárie, problemas periodontais e ortodônticos sejam maiores, pois, geralmente eles possuem dificuldades motoras e também apresentam uma menor tonicidade muscular da face. Além disso os cuidadores frequentemente oferecem alimentos macios e adocicados (Fonseca., 2018; Zink et al., 2018).

Alguns autores fizeram um estudo de revisão e não encontraram diferenças de patologias bucais em crianças com TEA. No entanto, as condições de higiene bucal e os problemas periodontais eram maiores quando comparados com crianças que não possuíam o transtorno. Além disso, foi observado uma maior prevalência de algumas desordens como hipoplasia de esmalte, mal oclusões, bruxismo, hábitos deletérios, gengivite e/ou doença periodontal e traumas dentários associados ao TEA (Chadah et al., 2012).

A maioria dos pacientes com transtorno do espectro autista possui um estado de saúde bucal deficiente, requerendo assim um acompanhamento odontológico mais frequente do que pacientes que não apresentem o transtorno. Assim, o profissional capacitado deve intervir da melhor maneira possível, sanando os problemas do paciente sem lhe causar traumas psicológicos (Mathu-Muju et al., 2016).

\section{O tratamento odontológico no paciente autista}

O ponto principal para o sucesso do tratamento odontológico, em pacientes autistas, é o conhecimento do profissional em relação à doença e o preparo para lidar com as suas características comportamentais. Também é importante que haja harmonia e confiança entre os pais/cuidadores e a equipe odontológica (Campos et al., 2009).

Para que o autista se familiarize com a equipe e o ambiente, muitas vezes é necessário mais de uma sessão. Deve-se procurar manter constância marcando sempre a consulta no mesmo dia e horário, além de manter sempre a mesma equipe (Zink et al., 2008).

O paciente portador do espectro autista tem dificuldade em manter o contato visual, e devido a isso o dentista deve utilizar meios para conseguir essa comunicação. O consultório deve ser calmo e tranquilo, o profissional deve ficar na mesma altura do paciente, com o objetivo de alcançar o contato visual, além de utilizar jalecos e gorros coloridos e óculos maiores (França., 2017). 
Podem ser aplicados diversos métodos para um domínio de comportamento. O método de TEACCH (Tratamento de educação para crianças autistas com Distúrbios Correlacionados à Comunicação), criado pelo Dr Eric Schopler em 1970, tem como objetivo desenvolver a independência da criança e organizar o seu espaço físico, através de uma rotina, utilizando diversos estímulos visuais, corporais e sonoros. O uso da comunicação alternativa pode auxiliar e facilitar o diálogo com esses pacientes, além de que este método pode ser realizado em diversas áreas da saúde (Yoshijinna., 2000)

Como estímulo visual, pode ser utilizada listas ilustrativas que contém imagens, descrevendo o passo a passo dos procedimentos que serão realizados durante a consulta. A técnica do 'Dizer-Mostrar-Fazer', onde se explica os procedimentos de preferência em alguém familiar, ajudando o portador do espectro a entender o que será realizado na consulta, é um exemplo de estímulo corporal. E nos estímulos sonoros são utilizados sons, palavras e verbos no imperativo, sempre de maneira tranquila e amigável (Yoshijinna., 2000).

A utilização de cartões de imagem que representam objetos, pessoas ou atividades, proporcionam treinamento e comunicação funcional. Este método busca ajudá-los a obter as coisas que desejam e auxilia na interação entre o profissional e o paciente (Sousa., 2015). Além disso, a utilização de aplicativos móveis também são uma ferramenta promissora que por meio de desenhos e áudios explicativos, influenciam positivamente a primeira consulta, eles ajudam a uma boa relação entre o dentista e o paciente, reduzindo a necessidade de possíveis intervenções mais invasivas, como o uso de anestesia geral (Oredugba et al., 2008; Busato et al., 2017).

Os pesquisadores Fakhruddin \& Batawi (2017) mencionaram em seu estudo a eficácia da distração áudio visual. Através da exibição de filmes projetados em tela, associados ao uso de óculos de realidade 3D, foi possível realizar exames clínicos e procedimentos odontológicos. Esse recurso associado a motivação do 'Falar-Mostrar-Fazer', mostrou ser uma excelente ferramenta para a redução do nível de ansiedade durante o tratamento, possibilitando a cooperação dos pacientes portadores de TEA (Zink et al., 2016).

O método de análise aplicada ao comportamento (ABA) também pode ser utilizado. Este exige que o profissional mantenha postura firme, e dê ordens para estimular o paciente a aprender habilidades que ainda não possui. Sempre que o paciente obedecer deverá receber elogios, ignorando os comportamentos indesejados (Amaral et al., 2012; Zink et al., 2016).

Já o método de restrição física tem como objetivo proteger a criança de possíveis materiais cortantes, que serão utilizados pelo profissional, para evitar injúrias durante o procedimento e é realizada com a ajuda dos pais e dos profissionais auxiliares. O paciente é envolvido por materiais como: lençóis, campos cirúrgicos, faixas com velcro, afim de evitar a movimentação tanto da cabeça como do corpo. A utilização de imobilização exige uma explicação clara ao paciente sobre o procedimento para que ele não o interprete como uma punição, sendo indispensável demonstrações de carinho e afeto com a criança. Esta técnica deve possuir autorização por escrito dos pais, afim de se evitar problemas éticos e legais futuros (Josgrilberg \& Cordeiro., 2005; Callahan et al., 2009; Shitsuka et al., 2015; Ferreira et al., 2016).

Em pacientes que apresentem extrema resistência ao tratamento odontológico dificultando o atendimento, e que os pais não aceitem o método de restrição física, podem ser utilizados meios de contenção química. São realizados por meio de sedação com óxido nitroso ou anestesia geral e recomenda-se o atendimento apenas do âmbito ambulatorial ou hospitalar devidamente equipado, com um profissional capacitado. (Marega \& Aiello., 2005; Sousa., 2015; Silva et al., 2016).

\section{Discussão}

O espectro autista foi definido pela Sociedade de Autismo da América (ASA) como uma complexa deficiência de desenvolvimento presente desde o nascimento, onde os primeiros sinais aparecem na primeira infância e afetam a capacidade de comunicação e interação com a sociedade (Amaral et al., 2012; Volpato et al., 2013., ASA., 2018). 
É um transtorno do neurodesenvolvimento que perdura por toda a vida e sua etiologia é múltipla e complexa que pode ser desencadeada pela interação de genes e fatores ambientais. Mesmo que este transtorno não tenha cura, estudos mostram que o diagnóstico e tratamentos precoces proporcionam maiores chances de minimizar os impactos negativos, possibilitando uma melhor qualidade de vida aos pacientes (Lord \& Schopler., 1985; Kessamiguiemon et al., 2017; Dell'Osso et al., 2018; Oliveira., 2018).

Com base na literatura percebe-se que ao passar do tempo ocorreu um aumento na quantidade de indivíduos diagnosticados com TEA. Apesar de não ter uma justificativa para este aumento, é certo que os profissionais da odontologia necessitam de preparo para atender estes pacientes, pois a tendência é o crescimento estatístico do número de casos. (Udhya $e t$ al., 2014; Zink et al., 2016)

O comportamento inesperado dos pacientes com TEA aliados com suas características próprias, como a dificuldade de relacionamento, agressividade, limitação motora e fonética, ansiedade (por estar em um ambiente diferente do habitual), são umas das maiores dificuldades que o profissional enfrenta no atendimento odontológico. Essas características complexas do transtorno favorecem para uma má higienização bucal destes pacientes (Katz et al., 2009).

Os pacientes autistas por apresentarem essas características comportamentais, são considerados por alguns autores propícios a doenças cariosas e periodontais. De acordo com a literatura fatores econômicos e sociodemográficos constituem forte influência na condição bucal dos portadores do espectro. Os estudos mostram que a cárie não é prevalente nesses indivíduos, mas podem haver maiores chances de surgirem, devido ao comprometimento motor, que implica em uma higiene bucal deficiente, além das medicações ingeridas diariamente (Oriqui \& Fett., 2006; Amaral., 2013; Villar et al., 2016).

Para obter um tratamento de sucesso desses pacientes, no consultório odontológico, é necessária a utilização de técnicas específicas para cada grau de autismo, atendendo as particularidades de cada indivíduo (Amaral et al., 2011; Gonçavez., 2012; Zink et al., 2016). Além disso é necessário que o cirurgião dentista e sua equipe sigam um protocolo para que o atendimento seja facilitado e para que o tratamento seja bem aceito pelo paciente. Um estudo mostrou que os especialistas em pediatria são profissionais mais bem preparados para atenderem os portadores de TEA, pois além de possuírem um melhor treinamento para lidarem com crianças na primeira infância, também são treinados para atenderem pacientes especiais (Katz et al., 2009; Udhya et al., 2014).

Por crianças autistas possuírem facilidade de processamento visual, os cirurgiões dentistas podem utilizar ferramentas para melhorar a comunicação com esses pacientes (Wibisono et al., 2009). A utilização de filmes, cartões de imagem, aplicativos móveis aliados a técnica "falar, mostrar e fazer" reduzem a ansiedade dos pacientes, o estresse no atendimento e permitem a realização de procedimentos odontológicos de modo menos traumático (Munaretto., 2002; Fakhruddin et al., 2017).

Para pacientes que não aderirem aos métodos citados acima, podem ser utilizados métodos de restrição física, sedação com óxido nitroso ou anestesia geral, onde todos devem possuir autorização por escrito dos pais a fim de evitar problemas éticos e legais. O primeiro visa proteger a crianças de materiais cortantes, além de ajudar o profissional a realizar o procedimento. Este deve ser bem esclarecido para a criança para que não seja mal interpretado como punição ou maus tratos. Já o segundo e o terceiro são utilizados nos casos em que os pais não autorizem a contenção ou o paciente não aceite, e são utilizados em âmbito hospitalar ou ambulatorial bem equipados e com profissionais capacitados. A imobilização também é utilizada nos casos onde não existam a possibilidade de sedação (Silva et al., 2016; Schardosim et al, 2015; Andrade \& Eleutélio., 2015; Ferreira et al., 2016).

\section{Considerações Finais}

Diante das inúmeras dificuldades encontradas pelos pais e cuidadores, os pacientes portadores do transtorno do espectro autista devem receber um tratamento interdisciplinar, onde o cirurgião-dentista pertencente a esta equipe deve transmitir a 
importância dos cuidados preventivos em relação as patologias bucais e as orientações quanto à dieta e higiene bucal, bem como o tratamento adequado. Para isso, o dentista que exerce papel fundamental no acompanhamento destes indivíduos, necessitam de capacitação e treinamento para torna-los aptos a prestar atendimento humanizado, por meio das técnicas de abordagem e protocolos específicos, com o objetivo de entender o paciente, compreender suas limitações e individualidades a fim de alcançar melhoria na qualidade de vida dessas famílias.

Ressaltamos, por fim, como sugestão para trabalhos futuros, que sejam abordados os aspectos mais específicos da condição bucal do autista e as diversas abordagens de tratamento, além de trabalhos clínicos e epidemiológicos.

\section{Referências}

Amaral, C. O. F., Malacrida, V. H., Videira, F. C. H., Parizi, A. G. S., de Oliveira, A. \& Straioto, F. G. Paciente autista: métodos e estratégias de condicionamento e adaptação para o atendimento odontológico. Archives of Oral Research. 2012;8(2):143-51.

Amaral, L. D. Comportamento de profissionais de saúde e familiares na abordagem integral das necessidades da saúde bucal de autistas em São José do Rio Preto, 2013.

Amaral, L. D., Portilho, J. A. C. \& Mendes, S. C. T. Estratégias de acolhimento e condicionamento do paciente autista na Saúde Bucal Coletiva. Revista Tempus - Actas de Saúde Coletiva, v. 5, n. 3, p. 105-114, 2011

American Psychiatric Association. Diagnostic and statistical manual of mental disorders. 5 ed. Washington: American Psychiatric Association; 2013.

American Psychiatry Association - APA. Manual diagnóstico e estatístico de 17 transtornos mentais. Tradução de Maria Inês Corrêa Nascimento et al. 5. ed. Porto Alegre: Artmed, 2014. 948 p.

Andrade, A. P. P., Eleutéio, A. S. L. Pacientes portadores de necessidades especiais: abordagem odontológica e anestesia geral. Revista Brasileira de Odontologia. 2015;72(1-2):66-9.

Arvidsson, O., Gillberg, C., Lichtenstein, P., et al. Alterações seculares no nível de sintomas do autismo diagnosticado clinicamente. J. Child. Psychol. Psiquiatria (2018).

Autism Society Of America - ASA. What is autism? Publicado em 2016. <www.autism-society.org/what-is/>.

Baio, J., Wiggins, L., Christensen, D. L., et al. Prevalence of Autism Spectrum Disorder Among Children Aged 8 Years — Autism and Developmental Disabilities Monitoring Network, 11 Sites, United States, 2014. Morbidity and Mortality Weekly Report Surveillance Summaries, v. 67, n. 6, p. 1-23, 2018.

Baronio, D., Castro, K., Gonchoroski, T., et al. Efeitos de um antagonista H3R no modelo animal de autismo induzido pela exposição pré-natal ao ácido valpróico. (2015) PLoS ONE 10: e0116363. 10.1371 / journal. pone.0116363

Bosa, C, A. Autismo: intervenções psicoeducacionais. Revista Brasileira de Psiquiatria. 2006;28:s47-s53.

Busato, P., Garbín, R. R., Nascimento Santos, C. N., Paranhos, L. R., Rigo, L..Influence of maternal anxiety on infant anxiety during dental care: a crosssectional study. São Paulo Medical Journal. São Paulo. abr, 2017.

Callahan, K., Mehta, S., Magee, S., \& Wie, M. (2009). ABA Versus TEACCH: The Case for Defining and Validating Comprehensive Treatment Models in Autism. Journal of autism and developmental disorders. 40. 74-88. 10.1007/s10803-009-0834-0.

Campos, C., Frazão, B., Saddi, G., Morais, L., Ferreira, M., Setúbal, P., et al. Manual prático para o atendimento odontológico de pacientes com necesidades especiais. Goiânia: Universidade Federal de Goiás; 2009

Chadha, G. M., Kakodkar, P., Chaugule, V., \& Nimbalkar, V. Dental survey of institutionalized children with autistic disorder. International Jornal of Clinical Pediatric Dentristy, v. 5, n. 1, p. 29-32, 2012

Dell'Osso, L., Abelli, M., Pini S, Carpita, B., Carlini, M., Mengali, F., et al. A influência do gênero nos sintomas do espectro da ansiedade social em uma amostra de estudantes universitários. Riv Psichiatr (2015) 50 (6 ): 295-301. 10.1708 / 2098.22688

Dell'Osso, L., Carpita, B., Cremone, I. M., Muti, D., Diadema, E., Barberi, F. M., et al. O efeito mediador do trauma e sintomas relacionados ao estressor e ruminações na relação entre traços autistas e espectro de humor. Psychiatry Res (2018). 10.1016 / j. psychres.2018.10.040

Fakhruddin, k. S., \& Batawi, H. Y. El. Effectiveness of audiovisual distraction in behavior modification during dental caries assessment and sealant placement in children with autism spectrum disorder. Dental Research Journal, Isfahan, v. 14, n. 3, p. 177-182, 2017.

Ferreira, R.., Oliveira, V., Piemonte, M.R.., Ramires, M. A.., Bruzamolin, C. D., \& Marques, F.R. Use of physical restraint as management techniques in odontologico babies treatment: Review literature. Revista Gestão \& Saúde. v. 14, n.1, p 31- 36, 2016.

Fonseca, A. L. A., Azzalis, L. A., Fonseca, F. L. A., \& Botazzo, C. Análise Qualitativadas percepções de cirurgiões-dentistas envolvidos nos atendimentos de pacientes com necessidades especiais de serviços públicos municipais. Rev. Bras. Crescimento Desenvolvimento Hum. 2010; 20 (2): 208-216.

Fonseca, V. Manual de Observação Psicomotora - Significação Psiconeurológica dos Factores Psicomotores.Lisboa: Âncora Editora, 2012. 
França, M. T. B. Transtorno de déficit de atenção e hiperatividade (TDAH): ampliando o entendimento. Jornal de Psicanálise; 45(82):191-207, <http://pepsic.bvsalud.org/scielo. php?script=sci_arttext\&pid=S0103-58352012 000100014>.

Gomes, P. T., Lima, L. H., Bueno, M. K., et al. Autism in Brazil: asystematic review of family challenges and coping strategies. Jornal de pediatria. 2015;91(2):111-21.

Gonçalves, J. B. Atendimento Odontológico à Pacientes com Necessidades Especiais: Uma revisão de Literatura. 2012.23 f. Trabalho de Conclusão de Curso (Especialização) - Faculdade de Medicina, Universidade Federal de Minas Gerais, Conselheiro Lafaiete - MG, 2012.

Josgrilberg, E. B., \& Cordeiro, R. L. Aspectos psicológicos do paciente infantil no atendimento de urgência. Odontol. clín.-cient, v. 4, n. 1, p. 13 -17, 2005. Kanner, L. Autistic disturbances of affective contact. publisher not identified, 1943.

Katz, C. R. T., Vieira, A., Meneses, J. M. L. P., \& Colares, V. Abordagem psicológica do paciente autista durante o atendimento odontológico. Odontol. clín.cient, v. 8, n. 2, p. 115-121, 2009.

Kessamiguiemon, V. G. G., Oliveira, K. D. C., \& Brum, S. C. TEA - Atendimento odontológico: relato de caso. Revista Pró-UniverSUS. 2017 Jul./Dez.; 08 (2): $67-71$.

Lord, C. \& Schopler, E. (1985). Differences in sex ratio in autism as a function of measured intelligence. Journal of Autism and Developmental Disorders, 15 , 185-193.

Maenner, M. J., Shaw, K. A., Baio, J., et al. Prevalência de Transtorno do Espectro do Autismo entre Crianças de 8 anos - Rede de Monitoramento de Deficiências de Desenvolvimento e Autismo, 11 Sites, Estados Unidos, 2016. MMWR Surveill Summ 2020; 69 (No. SS-4): 1-12.

Marega, T., \& Aiello, A. L. R. Autismo e tratamento odontológico: algumas considerações. JBP rev. Ibero-am. odontopediatr. odontol. bebê, v. 8, n. 42, p. $150-157,2005$

Mathu-Muju, K. R.., Li, H. F., Nam, L. H., \& Bush, H. M. Visualizing the Comorbidity Burden in Children with Autism Spectrum Disorder Receiving Dental Treatment Under General 18 Anesthesia. Pediatric Dentistry, v. 38, n. 2, p. 134-139, 2016.

Mello, A. M. S. R. Autismo: guia prático. 6. ed. São Paulo: AMA; Brasília: Corde, 2007, 104 p.

Munaretto, V. C. M. O autista e o tratamento odontológico.Goiânia (GO): Associação Brasileira de Odontologia seção Goiás. Especialização em Odontopediatria, 2002.

Oliveira, C. Um retrato do autismo no Brasil. Revista Espaço Aberto, 170. ed. São Paulo: Comunidade USP, 2015. <www.usp.br/espacoaberto/?materia=umretrato-do-autismo-no-brasil>.

Oredugba, F. A., Akindayomi, Y. Oral health status and treatment needs of children and young adults attending a day centre for individuals with special health care needs. BMC Oral Health, 2008.

Oriqui M. S. Y., \& Fett, C. A. Avaliação Clínica das Condições de Saúde Bucal de Pacientes Autistas. São José do Rio Preto. (Dissertação de Mestrado) Fac. de Medicina de São José do Rio Preto. 2006; 14-82.

Perez, E., Behar-horenstein, S., \& Guelmann, M. Crown-root Fracture Restoration on a Patient with Autism Spectrum Disorder. The Journal of Contemporary Dental Practice, v. 17, n. 9, p. 769-773, 2016.

Predebon, A., Darold, F.F., Volpato, S., \& Gallon, A. Método educacional para autistas: Reforço alternativo para o tratamento odontológico utilizando sistema de comunicação por figuras. Ação Odonto [Internet]. 2013; 1(1):[85-98 pp.]. file:///C:/Users/claudia/Downloads/3792-13430-1-PB\%20(1).pdf.

Sant'Anna, L. F. C., Barbosa, C. C. N., \& Brum, S. C. Atenção à saúde bucal do paciente autista. Revista Pró-UniverSUS. 2017 jan./jun.; 08 (1): $67-74$.

Santos, A. A., \& Fronza, C. A. A fala de uma criança autista dos 10 aos 11 anos de idade. Revista Entrelinhas, 2011; 5(2):55-57.

Schardosim, L. R., Costa J. R. S., \& Azevedo, M. S. Abordagem odontológica de pacientes com necessidades especiais em um centro de referência no sul do Brasil. Revista da AcBO. 2015;4(2).

Schimidt, C., Dell'aglio, D. D, \& Bosa, C.A. Estratégias de coping de mães de portadores de autismo lidando com dificuldades e com a emoção. Psicologia: Reflexão e Crítica. 2007; 20:124-131

Shitsuka, R. I. M., Shitsuka, C, Moriyama, C. M., Corrêa, F. N. P., Delfino, C. S., \& Corrêa, M. S. N. P. Development and assessment of the effectiveness of protective stabilization in pediatric dentistry: a pilot study. RFO.Passo Fundo, v. 20, n. 1, p. 59-63, jan./abr. 2015.

Silva, L.F.P., Freire, N.C, Santana, R.S., \& Miasato, J.M. Behavioral Management Techniques non Pharmacological in Pediatric Dentistry. Rev. Odontologia. Univ. Cid. São Paulo 2016; 28(2): 135-42,mai-ago

Sousa, P. L. Estratégias para a abordagem do paciente com perturbação do espectro do autismo na Medicina Dentária. 2015.

Souza, T.N., Sonegheti, J. V., Andrade, L. H. R., \& Tannure, P. N. Atendimento odontológico em uma criança com transtorno do espectro autista: relato de caso. 2017

Tarelho, L. G. Investigação da percepção dolorosa em pacientes com autismo de alto funcionamento. Dissertação de mestrado. Faculdade de Medicina da Universidade de São Paulo. São Paulo, 2005. 
Research, Society and Development, v. 10, n. 14, e496101422317, 2021

(CC BY 4.0) | ISSN 2525-3409 | DOI: http://dx.doi.org/10.33448/rsd-v10i14.22317

Udhya, J., Varadharaja, M. M., Parthiban, J., \& Ila, S. Autism Disorder (AD): An Updated Review for Paediatric Dentists. Journal of Clinical \& Diagnostic Research, v. 8, n. 2, p. 275-279, 2014.

Villar, B. B., Mourelle-Martínez, M. R., Diéguez-Pérez, M., et al. Incidence of oral health in paediatric patients with disabilities: Sensory disorders and autism spectrum disorder. Systematic review II. Journal of Clinical and Experimental Dentistry, v. 8, n. 3, p. e344-e351, 2016.

Volpato, S., Predebon, A., Darold, F. F., \& Gallon, A. Método educacional para autistas: Reforço alternativo para o tratamento odontológico utilizando sistema de comunicação por figuras. Ação Odonto, v. 1, n. 1, p. 85-98, 2013

Weddell, J. A, Sanders, B. J, \& Jones, J. E. Problemas odontológicos em crianças com necessidades especiais. In: McDonald RE, Avery DR, editors. Odontopediatria para crianças e adolescentes. 9 ed. Rio de Janeiro: Elsevier; 2011. p. 456-82.

Wibisono, W. L., Suharsini, M., Wiguna, T., Sudiroatmodjo, B., Budiardjo, S. B., \& Auerkari, E. I. Perception of dental visit pictures in children with autism spectrum disorder and their caretakers: A qualitative study. Journal of International Society of Preventive \& Community Dentistry, v. 6, n. 4, p. 359-365, 2016.

Yoshijinna, M. M. Autismo: orientação para pais. Casa do autista- Brasília: Ministério da Saúde, p. 38, 2000

Zink, A. G., Diniz, M. B., Santos, M. T. R., \& Guaré, R. O. Use of a Picture Exchange Communication System for preventive procedures in individuals with autism spectrum disorder: pilot study. Special Care in Dentistry, v. 36, n. 5, p. 254-259, 2016.

Zink, A. G., Molina, E. C., Diniz, M. B., Santos, M. T. B. R., \& Guaré, R. O. Communication application for use during the first dental visit for children and adolescents with autism spectrum disorders. Pediatric dentistry, v. 40, n. 1, p. 18-22, 2018.

Zink, A.G.; et al. Atendimento odontológico do paciente autista - Relato de caso. Revista ABO Nacional - vol.16, n5, 313-316, out/Nov, 2008. 